\title{
A MULTIVALUED FIXED POINT RESULT WITH ASSOCIATED DATA DEPENDECE AND STABILITY STUDY
}

\begin{abstract}
In this paper, our primary result is on the existence of non-empty fixed point sets for a new multivalued function defined here. An admissibility condition is also postulated and used in the main theorem. In two separate sections, we present data dependence and stability results for the non-empty fixed-point sets of these mappings. Some consequences of the main theorem are discussed. The analysis is in the most general setting of metric spaces. An illustrative example is discussed, which shows that our existence theorem effectively extends some results in this line of research.
\end{abstract}

Key words: fixed point, data dependence, stability, metric space, admissibility condition

2020 Mathematical Subject Classification: 47H10, 54H25, $54 H 10$

1. Introduction and Mathematical Background. Multivalued contractions appeared first in fixed point theory in the work of Nadler [14]. This work was followed by a development of the branch of fixed point theory in the domain of set-valued analysis. The books [2], [12] describe this development to a considerable extent.

There have been several generalizations of the famous Banach's contraction mapping principle, which appeared in the famous work of Banach [3] in 1922. Some of the generalizations are Boyd et al. [5] in 1969, Meir et al. [13] in 1969, Suzuki [18] in 2008, etc. This domain of mathematics is active in contemporary research as well. Also, many of these results have important applications in different problem of mathematics.

One of the recent generalizations is due to Feng and Liu [8] in 2006, which was followed by the introductions of several single and multivalued contractions, incorporating the basic idea of Feng and Liu. These (C) Petrozavodsk State University, 2022 
have come to be known as Feng-Liu-type contractions, which occupy an important place in metric fixed point theory.

Alongside, there has been another development in fixed point theory, which addresses the fact coming out of the observations: that in many established fixed point results the contraction condition between arbitrary pairs of points in the space is not utilized. There are scopes of restricting the domains of these contractions. Two approaches appeared for that, one is introduction of order relations on the spaces [9], [11], [15], and the other is the use of admissibility conditions, which are certain restrictions on the concerned mapping [1], [10], [17].

In this paper, we introduce a new multivalued contraction of Feng-Liu-type and a new admissibility condition. We utilize these concepts to prove a new result in fixed point theory. Further, we investigate the associated data dependence and stability problem of the fixed point sets. Some references on these topics are [4], [6], [7], [16].

We begin with the essentials of mathematics required for our purpose. Let $(X, \rho)$ be a metric space. We denote the collection of nonempty subsets of $X$ by $\mathcal{P}(X)$, the collection of nonempty bounded subsets of $X$ by $\mathcal{B}(X)$, and the collection of nonempty closed and bounded subsets of $X$ by $\mathcal{C B}(X)$.

Let $z \in X$ and $A \in \mathcal{B}(X)$. The distance of $A$ from $z$, denoted by $\mathcal{D}(z, A)$, is defined as $\mathcal{D}(z, A)=\inf \{\rho(z, y): y \in A\}$. The distance between two subsets $A, B \in \mathcal{C B}(X)$, denoted by $\mathcal{H}(A, B)$, is defined as $\mathcal{H}(A, B)=\max \left\{\sup _{z \in A} \mathcal{D}(z, B), \sup _{x \in B} \mathcal{D}(x, A)\right\} . \mathcal{H}$ is known as the Hausdorff metric induced by the metric $\rho$ on $\mathcal{C B}(X)$ [14]. Further, if $(X, \rho)$ is complete then $(\mathcal{C B}(X), \mathcal{H})$ is also complete.

Definition 1. A point $x \in X$ is called a fixed point of a multivalued mapping $T: X \rightarrow \mathcal{P}(X)$ if $x \in T x$. We denote the set of fixed points of $T$ by $F_{T}$.

Definition 2. A function $f: X \rightarrow \mathbb{R}$, where $(X, \rho)$ is a metric space, is said to be lower semi-continuous if $f(x) \leqslant \liminf _{n \rightarrow+\infty} f\left(x_{n}\right)$ for any convergent sequence $\left\{x_{n}\right\}$ in $X$ with limit $x \in X$.

Definition 3. [17] A mapping $f: X \rightarrow X$ is said to be $\eta$-admissible, where $\eta: X \times X \rightarrow[0,+\infty)$, if $\eta(u, v) \geqslant 1$, for $u, v \in X$ implies $\eta(f u, f v) \geqslant 1$.

In a separate vein, the following definition was introduced in [1]. 
Definition 4. [1] Let $X$ be a nonempty set and $\eta, \beta: X \rightarrow[0,+\infty)$. A function $f: X \rightarrow X$ is called a cyclic $(\eta-\beta)$-admissible mapping if

(i) $\eta(x) \geqslant 1$, for some $x \in X$, implies $\beta(f x) \geqslant 1$,

(ii) $\beta(x) \geqslant 1$, for some $x \in X$, implies $\eta(f x) \geqslant 1$.

In the following, we define cyclic $(\eta-\beta)$ admissibility for multivalued mappings.

Definition 5. Let $X$ be a nonempty set and $\eta, \beta: X \rightarrow[0,+\infty)$, $f: X \rightarrow \mathcal{P}(X)$. We say that $f$ is a cyclic $(\eta-\beta)$-admissible mapping if

(i) $\eta(x) \geqslant 1$, for some $x \in X$, implies $\beta(u) \geqslant 1$, for $u \in f x$,

(ii) $\beta(x) \geqslant 1$, for some $x \in X$, implies $\eta(u) \geqslant 1$, for $u \in f x$.

Example. Define $T: X \rightarrow \mathcal{P}(X)$, where $X=[0,1]$ is the usual metric space, as $T x=\left[0, \frac{x}{8}\right]$. Define $\eta, \beta: X \rightarrow[0,+\infty)$ as

$$
\eta(x)=\left\{\begin{array}{ll}
e^{x}, & \text { if } 0 \leqslant x \leqslant \frac{1}{2}, \\
0, & \text { otherwise }
\end{array} \text { and } \beta(x)= \begin{cases}\cosh x, & \text { if } 0 \leqslant x \leqslant \frac{1}{2} \\
0, & \text { otherwise }\end{cases}\right.
$$

Let $x \in X$ and $\eta(x) \geqslant 1$. Therefore, $x \in\left[0, \frac{1}{2}\right]$ and $T x=\left[0, \frac{x}{8}\right] \subseteq\left[0, \frac{1}{2}\right]$. Then $\beta(u) \geqslant 1$, for all $u \in T x$. Similarly, if $y \in X$ and $\beta(y) \geqslant 1$, it can be shown that $\eta(v) \geqslant 1$, for all $v \in T y$. Therefore, $T$ is a cyclic $(\eta-\beta)$-admissible mapping.

In the following, we define a generalized Feng-Liu-type contraction for multivalued mapping.

Definition 6. Let $(X, \rho)$ be a metric space, $T: X \rightarrow \mathcal{C B}(X)$ be a multivalued mapping and $\eta, \beta: X \rightarrow[0,+\infty)$. Let $b, c \in(0,1)$ with $c<b$. Then $T$ is said to be a generalized Feng-Liu-type contraction if for $x \in X$ with $\Delta(x) \geqslant 1$ there is a $y \in T x$, such that

$$
\begin{gathered}
b \rho(x, y) \leqslant f x \text { and } f y \leqslant c M(x, y), \text { where } \\
M(x, y)=\max \left\{\rho(x, y), \frac{\mathcal{D}(x, T x)+\mathcal{D}(y, T y)}{2}, \frac{\mathcal{D}(x, T y)}{2}\right\}, \\
\Delta(x)=\eta(x) \text { or } \beta(x) \text { and } f: X \rightarrow \mathbb{R} \text { is given by } f x=\mathcal{D}(x, T x), x \in X .
\end{gathered}
$$

Definition 7. Let $(X, \rho),\left(Y, \rho_{1}\right)$ be two metric spaces and $\mathcal{H}$ be the Hausdorff metric on $\mathcal{C B}(Y)$. A multivalued mapping $T: X \rightarrow \mathcal{C B}(Y)$ is 
said to be continuous at $z \in X$ if for any sequence $\left\{z_{n}\right\}$ in $X$, $\lim _{n \rightarrow+\infty} \mathcal{H}\left(T z, T z_{n}\right)=0$, whenever $\rho\left(z, z_{n}\right) \rightarrow 0$ as $n \rightarrow+\infty$.

\section{Multivalued fixed point results.}

Theorem 1. Let $T: X \rightarrow \mathcal{C B}(X)$ be a multivalued mapping and $\eta$, $\beta: X \rightarrow[0,+\infty)$, where $(X, \rho)$ is a complete metric space. Suppose that: (i) there exists $x_{0} \in X$, such that $\eta\left(x_{0}\right) \geqslant 1$ or $\beta\left(x_{0}\right) \geqslant 1$; (ii) $T$ is cyclic $(\eta-\beta)$-admissible; (iii) $T$ is continuous, or $f$, where $f(x)=\mathcal{D}(x, T x)$ for all $x \in X$, is lower semi-continuous; (iv) there exist $b, c \in(0,1)$ with $c<b$, such that $T$ is a generalized Feng-Liu-type contraction. Then $F_{T}$ is non-empty.

Proof. Since $T x \in \mathcal{C B}(X)$, for $x \in X$ and $b \in(0,1)$, we have the following: for any $x \in X$ there exists $y \in T x$, such that $\rho(x, y) \leqslant \frac{1}{b} \mathcal{D}(x, T x)$, that is,

$$
b \rho(x, y) \leqslant \mathcal{D}(x, T x)=f(x) .
$$

By the assumption (i), there exists $x_{0} \in X$, such that $\eta\left(x_{0}\right) \geqslant 1$ (the proof is similar if $\left.\beta\left(x_{0}\right) \geqslant 1\right)$. Then $\Delta\left(x_{0}\right) \geqslant 1$ and, hence, by (1) and the assumption (iv), we choose $x_{1} \in T x_{0}$ such that

$$
b \rho\left(x_{0}, x_{1}\right) \leqslant f x_{0} \text { and } f x_{1} \leqslant c M\left(x_{0}, x_{1}\right) .
$$

As $\eta\left(x_{0}\right) \geqslant 1$ and $x_{1} \in T x_{0}$, by the assumption (ii), we have $\beta\left(x_{1}\right) \geqslant 1$ and, hence, $\Delta\left(x_{1}\right)=\beta\left(x_{1}\right) \geqslant 1$. Then, by (1) and the assumption (iv), we choose $x_{2} \in T x_{1}$ such that

$$
b \rho\left(x_{1}, x_{2}\right) \leqslant f x_{1} \text { and } f x_{2} \leqslant c M\left(x_{1}, x_{2}\right) .
$$

As $\beta\left(x_{1}\right) \geqslant 1$ and $x_{2} \in T x_{1}$, by the assumption (ii), we have $\eta\left(x_{2}\right) \geqslant 1$ and, hence, $\Delta\left(x_{2}\right)=\eta\left(x_{2}\right) \geqslant 1$. Then, by (1) and the assumption (iv), we choose $x_{3} \in T x_{2}$ such that

$$
b \rho\left(x_{2}, x_{3}\right) \leqslant f x_{2} \text { and } f x_{3} \leqslant c M\left(x_{2}, x_{3}\right) .
$$

As $\eta\left(x_{2}\right) \geqslant 1$ and $x_{3} \in T x_{2}$, by the assumption (ii), we have $\beta\left(x_{3}\right) \geqslant 1$ and, hence, $\Delta\left(x_{3}\right)=\beta\left(x_{3}\right) \geqslant 1$. Then, by (1) and the assumption (iv), we choose $x_{4} \in T x_{3}$ such that

$$
b \rho\left(x_{3}, x_{4}\right) \leqslant f x_{3} \text { and } f x_{4} \leqslant c M\left(x_{3}, x_{4}\right) .
$$


As $\beta\left(x_{3}\right) \geqslant 1$ and $x_{4} \in T x_{3}$, by the assumption (ii), we have $\eta\left(x_{4}\right) \geqslant 1$ and, hence, $\Delta\left(x_{4}\right)=\eta\left(x_{4}\right) \geqslant 1$. In this way, we construct a sequence $\left\{x_{n}\right\}$, such that

$$
x_{n+1} \in T x_{n} \text { with } \eta\left(x_{2 n}\right) \geqslant 1, \beta\left(x_{2 n+1}\right) \geqslant 1 \text {, for all } n \geqslant 0 .
$$

By (2), either $\eta\left(x_{n}\right) \geqslant 1$ or $\beta\left(x_{n}\right) \geqslant 1$ for all $n \geqslant 0$ and, hence, $\Delta\left(x_{n}\right) \geqslant 1$, for all $n \geqslant 0$. Applying the assumption (iv), we have

$$
b \rho\left(x_{n}, x_{n+1}\right) \leqslant f x_{n} \text { and } f x_{n+1} \leqslant c M\left(x_{n}, x_{n+1}\right), \text { for all } n \geqslant 0,
$$

where $f x_{n}=\mathcal{D}\left(x_{n}, T x_{n}\right)$ and

$$
\begin{gathered}
M\left(x_{n}, x_{n+1}\right)= \\
=\max \left\{\rho\left(x_{n}, x_{n+1}\right), \frac{\mathcal{D}\left(x_{n}, T x_{n}\right)+\mathcal{D}\left(x_{n+1}, T x_{n+1}\right)}{2}, \frac{\mathcal{D}\left(x_{n}, T x_{n+1}\right)}{2}\right\} \leqslant \\
\leqslant \max \left\{\rho\left(x_{n}, x_{n+1}\right), \frac{\rho\left(x_{n}, x_{n+1}\right)+\rho\left(x_{n+1}, x_{n+2}\right)}{2}, \frac{\rho\left(x_{n}, x_{n+2}\right)}{2}\right\}= \\
=\max \left\{\rho\left(x_{n}, x_{n+1}\right), \frac{\rho\left(x_{n}, x_{n+1}\right)+\rho\left(x_{n+1}, x_{n+2}\right)}{2},\right. \\
\left.\frac{\rho\left(x_{n}, x_{n+1}\right)+\rho\left(x_{n+1}, x_{n+2}\right)}{2}\right\} \leqslant \\
\leqslant \max \left\{\rho\left(x_{n}, x_{n+1}\right), \rho\left(x_{n+1}, x_{n+2}\right)\right\} .
\end{gathered}
$$

Suppose that $0 \leqslant \rho\left(x_{n}, x_{n+1}\right)<\rho\left(x_{n+1}, x_{n+2}\right)$. Then, from (3) and (4), we have

$$
\begin{aligned}
b \rho\left(x_{n+1}, x_{n+2}\right) & \leqslant f x_{n+1} \leqslant c M\left(x_{n}, x_{n+1}\right) \leqslant \\
& \leqslant c \max \left\{\rho\left(x_{n}, x_{n+1}\right), \rho\left(x_{n+1}, x_{n+2}\right)\right\} \leqslant c \rho\left(x_{n+1}, x_{n+2}\right) .
\end{aligned}
$$

As $c<b$, it is a contradiction. Thus, we have $\rho\left(x_{n+1}, x_{n+2}\right) \leqslant \rho\left(x_{n}, x_{n+1}\right)$, for all $n \geqslant 0$. Therefore, using (3) and (4), we come to

$$
\begin{aligned}
b \rho\left(x_{n}, x_{n+1}\right) & \leqslant \mathcal{D}\left(x_{n}, T x_{n}\right), \\
\mathcal{D}\left(x_{n+1}, T x_{n+1}\right) & \leqslant c \rho\left(x_{n}, x_{n+1}\right), \text { for all } n \geqslant 0 .
\end{aligned}
$$

From (2) and (5), we have

$$
\left.\begin{array}{c}
b \rho\left(x_{n+1}, x_{n+2}\right) \leqslant c \rho\left(x_{n}, x_{n+1}\right), \text { that is, } \rho\left(x_{n+1}, x_{n+2}\right) \leqslant \frac{c}{b} \rho\left(x_{n}, x_{n+1}\right) \\
\mathcal{D}\left(x_{n+1}, T x_{n+1}\right) \leqslant c \rho\left(x_{n}, x_{n+1}\right), \text { that is, } \mathcal{D}\left(x_{n+1}, T x_{n+1}\right) \leqslant \frac{c}{b} \mathcal{D}\left(x_{n}, T x_{n}\right) .
\end{array}\right\}
$$


By repeated application of the above inequalities, we get

$$
\left.\begin{array}{c}
\rho\left(x_{n+1}, x_{n+2}\right) \leqslant\left[\frac{c}{b}\right]^{n+1} \rho\left(x_{0}, x_{1}\right), \text { for all } n \in \mathbb{N}, \\
\mathcal{D}\left(x_{n+1}, T x_{n+1}\right) \leqslant\left[\frac{c}{b}\right]^{n+1} \mathcal{D}\left(x_{0}, T x_{0}\right), \text { for all } n \in \mathbb{N} .
\end{array}\right\}
$$

As $c<b$, we have $a=\frac{c}{b}<1$. Hence, $a^{n} \rightarrow 0$ as $n \rightarrow+\infty$. For $m, n \in \mathbb{N}$ with $m>n$, we have

$$
\begin{aligned}
\rho\left(x_{n}, x_{m}\right) & \leqslant \rho\left(x_{n}, x_{n+1}\right)+\rho\left(x_{n+1}, x_{n+2}\right)+\ldots+\rho\left(x_{m-1}, x_{m}\right) \leqslant \\
& \leqslant a^{n} \rho\left(x_{0}, x_{1}\right)+a^{n+1} \rho\left(x_{0}, x_{1}\right)+\ldots+a^{m-1} \rho\left(x_{0}, x_{1}\right) \leqslant \\
& \leqslant \sum_{k=n}^{+\infty} a^{k} \rho\left(x_{0}, x_{1}\right)=\frac{a^{n}}{(1-a)} \rho\left(x_{0}, x_{1}\right) \rightarrow 0 \text { as } n \rightarrow+\infty .
\end{aligned}
$$

Hence, $\left\{x_{n}\right\}$ is a Cauchy sequence. As $(X, \rho)$ is complete, there exists a point $x \in X$, such that

$$
x_{n} \rightarrow x \text { as } n \rightarrow+\infty \text {. }
$$

We consider the two following cases.

Case (i): Suppose that $T$ is continuous.

Using (8) and the continuity of $T$, we get $\mathcal{H}\left(T x_{n}, T x\right) \rightarrow 0$ as $n \rightarrow+\infty$, which implies that $\mathcal{D}\left(x_{n+1}, T x\right) \rightarrow 0$ as $n \rightarrow+\infty$, that is, $\mathcal{D}(x, T x)=0$. Since $T x \in \mathcal{C B}(X), T x=\overline{T x}$, where $\overline{T x}$ denotes the closure of $T x$. Now, $\mathcal{D}(x, T x)=0$ implies that $x \in \overline{T x}=T x$, that is, $x \in F_{T}$. Therefore, $F_{T}$ is non-empty.

Case (ii): Suppose that $f$, where $f(x)=\mathcal{D}(x, T x)$ for all $x \in X$, is lower semi-continuous.

As $\frac{c}{b}<1$, we have from (7) that $\lim _{n \rightarrow+\infty} f x_{n}=\lim _{n \rightarrow+\infty} \mathcal{D}\left(x_{n}, T x_{n}\right)=0$. Then $0 \leqslant \mathcal{D}(x, T x)=f x \leqslant \liminf _{n \rightarrow+\infty} f x_{n}=0$, that is, $\mathcal{D}(x, T x)=0$. Arguing as above, we get $x \in F_{T}$, that is, $F_{T}$ is non-empty.

3. Data dependence of fixed point sets. Here we prove a data dependence result for the mappings described in the previous section. The problem of data dependence is to estimate the distance between the fixed point set of an operator with the corresponding fixed point set of 
another mapping where the functional value of the mapping at every point is different from that of the given operator by a magnitude less than a given number. It is generally considered for multivalued operators. In that case, the Hausdorff distance is often used for estimating distance between two functional values, wherever it is applicable.

Here we investigate the data dependence of fixed point sets of the setvalued contractions mentioned in Section 2.

Theorem 2. Let $(X, \rho)$ be a complete metric space, $\eta, \beta: X \rightarrow[0,+\infty)$. Let $T_{1}, T_{2}: X \rightarrow \mathcal{C B}(X)$ be two multivalued mappings, such that $\mathcal{H}\left(T_{1} x, T_{2} x\right) \leqslant K$ for all $x \in X$, where $K>0$ is a fixed a real number. Suppose that $T_{2}$ satisfies the assumptions (ii), (iii) and (iv) of Theorem 1, $F_{T_{1}} \neq \emptyset$ and $\eta(x) \geqslant 1$ or $\beta(x) \geqslant 1$ for any $x \in F_{T_{1}}$. Then $F_{T_{2}} \neq \emptyset$ and $\sup _{x \in F_{T_{1}}} \mathcal{D}\left(x, F_{T_{2}}\right) \leqslant \frac{K}{b-c}$.

Proof. By the assumption of the theorem, $F_{T_{1}} \neq \emptyset$. Let $y_{0} \in F_{T_{1}}$, that is, $y_{0} \in T_{1} y_{0}$. Then $\eta\left(y_{0}\right) \geqslant 1$ or $\beta\left(y_{0}\right) \geqslant 1$. Therefore, we know that there exists $y_{0} \in X$, such that $\eta\left(y_{0}\right) \geqslant 1$ or $\beta\left(y_{0}\right) \geqslant 1$, and $T_{2}$ satisfies the assumptions (ii), (iii) and (iv) of Theorem 1. By Theorem 1, $F_{T_{2}}$ is nonempty, that is, $F_{T_{2}} \neq \emptyset$. Assuming that $\eta\left(y_{0}\right) \geqslant 1$ (the proof is similar if $\beta\left(y_{0}\right) \geqslant 1$ ) and arguing similarly as in the proof of Theorem 1 , we construct a sequence $\left\{y_{n}\right\}$, such that

$$
\begin{aligned}
y_{n+1} \in T_{2} y_{n} ; \quad \eta\left(y_{2 n}\right) \geqslant 1, \beta\left(y_{2 n+1}\right) & \geqslant 1, \text { for all } n \geqslant 0, \\
b \rho\left(y_{n}, y_{n+1}\right) \leqslant \mathcal{D}\left(y_{n}, T_{2} y_{n}\right), \quad \mathcal{D}\left(y_{n+1}, T_{2} y_{n+1}\right) & \leqslant c \rho\left(y_{n}, y_{n+1}\right), \text { for all } n \geqslant 0
\end{aligned}
$$

and

$$
\left.\begin{array}{c}
\rho\left(y_{n+1}, y_{n+2}\right) \leqslant\left[\frac{c}{b}\right]^{n+1} \rho\left(y_{0}, y_{1}\right), \text { for all } n \geqslant 0, \\
\mathcal{D}\left(y_{n+1}, T_{2} y_{n+1}\right) \leqslant\left[\frac{c}{b}\right]^{n+1} \mathcal{D}\left(y_{0}, T_{2} y_{0}\right), \text { for all } n \geqslant 0 .
\end{array}\right\}
$$

Like in the proof of Theorem 1, we prove $\left\{y_{n}\right\}$ to be a Cauchy sequence in $X$ and that there exists $u \in X$, such that

$$
y_{n} \rightarrow u \text { as } n \rightarrow+\infty
$$

and $u$ is a fixed point of $T_{2}$, that is, $u \in T_{2} u$. Following (10), we have

$$
\rho\left(y_{0}, y_{1}\right) \leqslant \frac{1}{b} \mathcal{D}\left(y_{0}, T_{2} y_{0}\right) \leqslant \frac{1}{b} \mathcal{H}\left(T_{1} y_{0}, T_{2} y_{0}\right) \leqslant \frac{K}{b} \text {. }
$$


Using (11), we have

$$
\rho\left(y_{0}, u\right) \leqslant \sum_{i=0}^{n} \rho\left(y_{i}, y_{i+1}\right)+\rho\left(y_{n+1}, u\right) \leqslant \sum_{i=0}^{n}\left[\frac{c}{b}\right]^{i} \rho\left(y_{0}, y_{1}\right)+\rho\left(y_{n+1}, u\right) .
$$

Taking limit as $n \rightarrow+\infty$ in the above inequality and using (12) and (13), we have

$$
\rho\left(y_{0}, u\right) \leqslant \sum_{i=0}^{+\infty}\left[\frac{c}{b}\right]^{n} \rho\left(y_{0}, y_{1}\right) \leqslant \frac{1}{\left(1-\frac{c}{b}\right)} \rho\left(y_{0}, y_{1}\right) \leqslant \frac{b}{(b-c)} \frac{K}{b} \leqslant \frac{K}{b-c} .
$$

Thus, given $y_{0} \in F_{T_{1}}$, we have $u \in F_{T_{2}}$, for which $\rho\left(y_{0}, u\right) \leqslant \frac{K}{b-c}$ holds. Hence, $\mathcal{D}\left(y_{0}, F_{T_{2}}\right) \leqslant \frac{K}{b-c}$. As $y_{0} \in F_{T_{1}}$ is arbitrary, we get $\sup _{x \in F_{T_{1}}} \mathcal{D}\left(x, F_{T_{2}}\right) \leqslant \frac{K}{b-c}$.

4. Stability of fixed point sets. In this section, we have a stability result, in which we answer the question whether for a convergent sequence of functions of the category considered in Section 2, the corresponding fixed point sets also converge to the fixed point set of the limiting function.

Theorem 3. Let $(X, \rho)$ be a complete metric space, $\eta, \beta: X \rightarrow[0,+\infty)$. Suppose there exists $x_{0} \in X$, such that $\eta\left(x_{0}\right) \geqslant 1$ or $\beta\left(x_{0}\right) \geqslant 1$. Let $\left\{T_{n}: X \rightarrow \mathcal{C B}(X): n \in \mathbb{N}\right\}$ be a sequence of continuous multivalued mappings, which uniformly converges to $T: X \rightarrow \mathcal{C B}(X)$. Suppose that each $T_{n}(n \in \mathbb{N})$ and $T$ satisfy the assumptions (ii) and (iv) of Theorem 1. Then $F_{T_{n}} \neq \emptyset$, for each $n \in \mathbb{N}$ and $F_{T} \neq \emptyset$. Furthermore, suppose that $\beta(x) \geqslant 1$ or $\eta(x) \geqslant 1$ for any $x$ belonging to $F_{T_{n}}(n \in \mathbb{N})$ and $F_{T}$. Then $\lim _{n \rightarrow+\infty} \mathcal{H}\left(F_{T_{n}}, F_{T}\right)=0$, that is, the fixed point sets of the sequence of mappings $\left\{T_{n}\right\}$ are stable.

Proof. Since each $T_{n}(n \in \mathbb{N})$ is continuous and $T_{n} \rightarrow T$ uniformly as $n \rightarrow+\infty, T$ is continuous. Then each $T_{n}(n \in \mathbb{N})$ and $T$ satisfy the assumptions (ii), (iii) and (iv) of Theorem 1. By Theorem 1, we have $F_{T_{n}} \neq \emptyset$, for each $n \in \mathbb{N}$ and $F_{T} \neq \emptyset$. Let $K_{n}=\sup _{x \in X} \mathcal{H}\left(T_{n} x, T x\right)$, where $n \in \mathbb{N}$. Since the sequence $\left\{T_{n}\right\}$ is uniformly convergent to $T$, we have

$$
\lim _{n \rightarrow+\infty} K_{n}=\lim _{n \rightarrow+\infty} \sup _{x \in X} \mathcal{H}\left(T_{n} x, T x\right)=0
$$


By Theorem 2, we have $\sup _{x \in F_{T_{n}}} \mathcal{D}\left(x, F_{T}\right) \leqslant \frac{K_{n}}{b-c}$ and $\sup _{x \in F_{T}} \mathcal{D}\left(x, F_{T_{n}}\right) \leqslant \frac{K_{n}}{b-c}$, for all $n \in \mathbb{N}$. Combining these two inequalities, we get

$$
\mathcal{H}\left(F_{T_{n}}, F_{T}\right) \leqslant \frac{K_{n}}{b-c}, \text { for all } n \in \mathbb{N} .
$$

Taking limit as $n \rightarrow+\infty$ in the above inequality and using (14), we have

$$
\lim _{n \rightarrow+\infty} \mathcal{H}\left(F_{T_{n}}, F_{T}\right) \leqslant \lim _{n \rightarrow+\infty} \frac{K_{n}}{b-c}=0
$$

that is, $\lim _{n \rightarrow+\infty} \mathcal{H}\left(F_{T_{n}}, F_{T}\right)=0$. So, the fixed point sets of the sequence of mappings $\left\{T_{n}: n \in \mathbb{N}\right\}$ are stable.

5. Consequences and illustration. We have the following consequent results of our main theorem.

Theorem 4. Let $(X, \rho)$ be a complete metric space and $T: X \rightarrow$ $\mathcal{C B}(X)$ be a multivalued mapping, such that $T$ is continuous or $f$, where $f(x)=\mathcal{D}(x, T x)$ for all $x \in X$ is lower semi-continuous. Suppose that there exists $c \in(0,1)$, such that for all $x, y \in X$

$$
\mathcal{H}(T x, T y) \leqslant c M(x, y) .
$$

Then $F_{T}$ is nonempty.

Proof. Let us define $\eta, \beta: X \rightarrow[0,+\infty)$ as $\eta(x)=\beta(x)=1$, for all $x \in X$. Then the assumptions (i) and (ii) of Theorems 1 are trivially satisfied. Let $x \in X$ and $b \in(0,1)$ with $c<b$. As $b \in(0,1)$, there exists $y \in T x$, such that $b \rho(x, y) \leqslant \mathcal{D}(x, T x)=f x$. Hence, by contractive inequality of the theorem, we have $f y=\mathcal{D}(y, T y) \leqslant \mathcal{H}(T x, T y) \leqslant c M(x, y)$. Therefore, for $x \in X$ with $\Delta(x) \geqslant 1$, there is a $y \in T x$, such that $b \rho(x, y) \leqslant f x$ and $f y \leqslant c M(x, y)$. Hence, by an application of Theorem 1, we conclude that $F_{T}$ is nonempty.

Theorem 5. [14] Let $(X, \rho)$ be a complete metric space and $T: X \rightarrow \mathcal{C B}(X)$ be a multivalued mapping. Suppose that there exists $c \in(0,1)$, such that for all $x, y \in X$,

$$
\mathcal{H}(T x, T y) \leqslant c \rho(x, y)
$$

Then $F_{T}$ is nonempty. 
Proof. The inequality of the theorem implies that mapping $T$ is continuous. Then the proof of the theorem (Nadler's result) follows from that of Theorem 4 .

Example. Take the metric space $(X, \rho)$, where $X=\{0,1,2, \ldots, n, \ldots\}$ and

$$
\rho(x, y)=\left\{\begin{array}{l}
0, \quad \text { if } \quad x=y \\
x+y, \quad \text { if } \quad x \neq y
\end{array}\right.
$$

Let $T: X \rightarrow \mathcal{C B}(X)$ be defined as $T(x)=\left\{\begin{array}{l}\{4,5\}, \quad \text { if } x=0,4,5, \\ \{2,3\}, \quad \text { if } x=1,2,3, \\ \{7\}, \quad \text { if } x=6,8, \\ \{8\}, \quad \text { if } x=7, \\ \{x, x+1, x+2\}, \quad \text { if } x \geqslant 9 .\end{array}\right.$

Define $\eta, \beta: X \rightarrow[0,+\infty)$ as $\eta(x)=\left\{\begin{array}{l}e^{x}, \text { if } x=0,1, \ldots, 5, \\ 0, \text { otherwise }\end{array}\right.$ and $\beta(x)=\left\{\begin{array}{l}2, \text { if } x=0,1, \ldots, 5 \\ 0, \text { otherwise }\end{array}\right.$

Take $b=0.7$ and $c=0.5$. Then all the conditions of Theorem 1 are satisfied and here $F_{T}=X-\{0,1,6,7,8\}$ is the fixed point set of $T$.

Remark 1. Let us take $x=0$ and $y=1$ in the example above. Then

$$
\mathcal{H}(T x, T y)=\mathcal{H}(T 0, T 1)=7>1=\rho(0,1)=\rho(x, y),
$$

which shows that there exists no $c \in(0,1)$, such that $\mathcal{H}(T x, T y) \leqslant$ c $\rho(x, y)$. Therefore, the inequality of Theorem 5 is not satisfied and, hence, Theorem 5 is not applicable to this example. So, Theorem 1 is a proper generalization of Theorem 5, that is, Theorem 5 in [14].

Remark 2. Let us take $x=6$ in the example above. Then $y=7$ is the only member in Tx and $b \rho(x, y)=0.7 \times 13=9.1<f(x)=D(x, T x)=13$. But $f(y)=D(y, T y)=15>13=\rho(x, y)$. There exists no $c \in(0,1)$, such that $f(y)=D(y, T y) \leqslant c \rho(x, y)$. Therefore, Theorem 3.1 in [8] is not applicable to this example.

Remark 3. The result of Feng and Liu (Theorem 3.1 of [8]) is derived for mappings that are from $X$ to $\mathcal{C}(X)$, where $\mathcal{C}(X)$ is the set of all closed subsets of $X$. Our result (Theorem 1) generalizes the result mentioned 
above for the case where the functional values are restricted to $\mathcal{C B}(X)$. The above Remark 2 shows that in this case the generalization is exact.

Acknowledgments. The authors gratefully acknowledge the suggestions made by the learned referee.

\section{References}

[1] Alizadeh S., Moradlou F., Salimi P. Some fixed point results for $(\alpha-\beta)$ $(\psi-\varphi)$ - contractive mappings. Filomat, 2014, vol. 28 , no. 3 , pp. $635-647$. DOI: $10.2298 /$ FIL1403635A

[2] Amar A. B., O'Regan D. Topological fixed point theory for singlevalued and multivalued mappings and applications. Springer International Publishing, Switzerland, 2016.

DOI: https://doi.org/10.1007/978-3-319-31948-3

[3] Banach S. Sur les opérations dans les ensembles abstraits et leurs applications aux équations intégrales. Fund Math., 1922, vol. 3, pp. 133-181.

[4] Bose R. K., Mukherjee R. N. Stability of fixed point sets and common fixed points of families of mappings. Indian J. Pure Appl. Math., 1980, vol. 11, no. 9, pp. $1130-1138$.

[5] Boyd D. W., Wong J. S. W. On nonlinear contractions. Proc. Amer. Math. Soc., 1969, vol. 20, pp. $458-464$.

DOI: https://doi.org/10.1090/S0002-9939-1969-0239559-9

[6] Chifu C., Petruşel G. Coupled fixed point results for $(\varphi, G)$-contractions of type (b) in b-metric spaces endowed with a graph. J. Nonlinear Sci. Appl., 2017, vol. 10, pp. $671-683$. DOI: 10.22436/jnsa.010.02.29

[7] Choudhury B. S., Metiya N., Kundu S. Existence, data-dependence and stability of coupled fixed point sets of some multivalued operators. Chaos, Solitons and Fractals, 2020, vol. 133.

DOI: $10.1016 / \mathrm{j}$.chaos.2020.109678.

[8] Feng Y., Liu S. Fixed point theorems for multi-valued contractive mappings and multi-valued Caristi type mappings. J. Math. Anal. Appl., 2006, vol. 317, pp. 103-112.

DOI: https://doi.org/10.1016/j.jmaa.2005.12.004

[9] Harjani J., Sadarangani K. Fixed point theorems for weakly contractive mappings in partially ordered sets. Nonlinear Anal., 2009, vol. 71, pp. 3403 -3410. DOI: https://doi.org/10.1016/j.na.2009.01.240

[10] Hussain N., Karapinar E., Salimi P., Akbar F. $\alpha$-admissible mappings and related fixed point theorems. J. Inequal. Appl., 2013, Article number: 114(2013). DOI: https://doi.org/10.1186/1029-242X-2013-114 
[11] Kadelburg Z., Radenović S. Fixed point and tripled fixed point theorems under Pata-type conditions in ordered metric spaces. International Journal of Analysis and Applications, 2014, vol. 6, no. 1, pp. 113-122.

[12] Kirk W., Shahzad N. Fixed point theory in distance spaces. Springer International Publishing, Switzerland, 2014.

DOI: https://doi.org/10.1007/978-3-319-10927-5

[13] Mier A., Keeler E. A theorem on contraction mappings. J. Math. Anal. Appl., 1969, vol. 28, pp. 326-329.

DOI: https://doi.org/10.1016/0022-247X (69)90031-6

[14] Nadler S. B. Jr. Multivalued contraction mapping. Pac. J. Math., 1969, vol. 30 , no. 2 , pp. $475-488$.

[15] Ran A. C. M., Reurings M. C. B. A fixed point theorem in partially ordered sets and some applications to matrix equations. Proc. Amer. Math. Soc., 2004, vol. 132, no. 5, pp. $1435-1443$.

DOI: https://doi.org/10.1090/S0002-9939-03-07220-4

[16] Rus I. A., Petruşel A., Sîntămărian A. Data dependence of the fixed point set of some multivalued weakly Picard operators. Nonlinear Anal., 2003, vol. 52, pp. 1947 -1959. DOI: 10.1016/S0362-546X (02) 00288-2

[17] Samet B., Vetro C., Vetro P. Fixed point theorems for $\alpha-\psi$-contractive type mappings. Nonlinear Anal., 2012, vol. 75, pp. 2154-2165.

DOI: https://doi.org/10.1016/j.na.2011.10.014

[18] Suzuki T. A generalized Banach contraction principle that characterizes metric completeness. Proc. Amer. Math. Soc., 2008, vol. 136, no. 5, pp. $1861-1869$.

DOI: https://doi.org/10.1090/S0002-9939-07-09055-7

Received June 1, 2021.

In revised form, October 18, 2021.

Accepted October 22, 2021.

Published online November 16, 2021.

Binayak S. Choudhury

Indian Institute of Engineering Science and Technology,

Shibpur, Howrah - 711103, West Bengal, India

E-mail: binayak12@yahoo.co.in, binayak@math.iiests.ac.in 
Nikhilesh Metiya

Sovarani Memorial College,

Jagatballavpur, Howrah-711408, West Bengal, India

E-mail: metiya.nikhilesh@gmail.com, nikhileshm@smc.edu.in

Sunirmal Kundu

Government General Degree College,

Salboni, Paschim Mednipur - 721516, West Bengal, India.

E-mail: sunirmalkundu2009@rediffmail.com 